\title{
A questionairre based study evaluating current medication adherence monitoring practice in a tertiary care teaching hospital
}

\author{
Nehal A Shah ${ }^{1 *}$, Vansh Nagrani ${ }^{2}$, Varsha Patel $^{3}$ \\ ${ }^{\mathbf{1}}$ Assistant Professor, ${ }^{2}$ Medical Student, ${ }^{3}$ Professor, Dept. of Pharmacology, Dr. M. K. Shah Medical College \& Research Centre, \\ Ahmedabad, Gujarat, India \\ *Corresponding Author: Nehal A Shah \\ Email: nehalsbks@gmail.com
}

\begin{abstract}
Introduction: Despite the high prevalence rates and negative impact of low medication adherence among patients, there is a paucity of information on current practices of clinicians in an Indian setting to monitor medication adherence.

Aim: The core aim of our study was to understand current clinical practice and perceptions about medication adherence monitoring.

Materials and Methods: This was a cross-sectional questionnaire based study. The questionnaire was distributed to 100 actively practicing clinicians across different levels at our tertiary care teaching Hospital. The questionnaire was structured to address the core objectives through 5 core questions and 10 sub questions related to medication adherence monitoring practice. Three questions were asked regarding their perceptions of medication adherence monitoring. Modified Likert scales were used to evaluate responses. Descriptive statistics like mean, standard deviation, frequencies and percentages were used to analyze data. Statistical tests like $\mathrm{t}$-test and $\mathrm{Chi}^{2}$ tests were used to examine group differences. Statistical significance was set at $\mathrm{p}<0.05$.

Results: Sixty-two (62\%) participants routinely monitor all patients for medication adherence. Most participants recognized themselves as responsible for medication adherence monitoring (79\%). On a scale of 1 to 10 , participants rated their adherence monitoring practices as an average of 7.01. The strategy most commonly classified as used "all the time" was asking patient about taking medications regularly (72\%). The strategies most commonly classified as "not at all" used were using electronic monitoring (73\%) and monitoring blood levels of the drug to assess adherence (59\%). These strategies were also rated the least effective. The strategies most commonly classified as "largely" effective included asking patients about problems or difficulties taking prescribed medications (55\%). Most clinicians believe other ancillary health care providers should be included in medication adherence related practices.
\end{abstract}

Conclusion: Our results demonstrate the need to develop systems supporting medication adherence monitoring in all hospitals.

Keywords: Medication adherence, Adherence monitoring, Current practice, Questionnaire.

\section{Introduction}

Medication adherence has been defined by the World Health Organization as "the extent to which a person's behaviortaking medication, following a diet, and/or executing lifestyle changes - corresponds with agreed recommendations from a health care provider." ${ }^{1}$ Previous studies have established the problems associated with poor adherence. ${ }^{2-4}$ Despite this, half of all patients, diagnosed with a chronic disease do not take medications as have been prescribed, with some studies reporting non adherence rates as high as $94 \% .^{1,5,6}$

It is important for hospitals to assess their own "practices and perceptions" of prescribers in relation to medication adherence monitoring. Detecting medication non-adherence among patients is a complex problem and clinicians form an integral part of the solution..$^{2,7-9}$ To the best of our knowledge, there is a paucity of data in this area.

Hence, the core aim of our study was to understand current clinical practice of clinicians working in different departments and at different experience levels in a tertiary care teaching hospital in regards to medication adherence monitoring.

\section{Objectives}

1. To identify current strategies of assessing medication adherence by clinicians.

2. To evaluate the effectiveness of these strategies from the perspective of the clinician.
3. To assess general perceptions of clinicians in regards to medication adherence monitoring.

4. To assess impact of clinical experience and training in medication adherence on medication adherence monitoring practice.

\section{Materials and Methods}

The study was conducted at SMS Multi-Specialty HospitalAhmedabad, a tertiary care teaching hospital. Prior permission for carrying out the study was obtained from the Institutional Ethics Committee.

\section{Design and study population}

This was a cross-sectional questionnaire based study. The questionnaire was adapted from studies conducted by Patel et al., Berben et al. and Maddux et al. ${ }^{7-9}$ The questionnaire was distributed to all clinicians across different levels (including Professors, Associate Professors, Assistant Professors, Senior Residents \& Junior Residents) at our Hospital. Our sample size was 100 participants. The questionnaire was pre-validated.

\section{Questionairre}

The questionnaire was structured to address the objectives through 5 core questions and 10 sub questions related to medication adherence monitoring practice. Three questions were asked regarding perceptions of medication adherence monitoring. 
For the medication adherence monitoring strategies, participants were asked to indicate on a 4-point scale the frequency (not at all, sometimes, all the time, would like to but not feasible) and on a 3-point scale the effectiveness of the intervention in their estimate (not at all, somewhat or largely).

Only participants actively practicing at our hospital, having treated patients in the month prior to study participation and with M.B;B.S/BDS degree with or without a post graduate (MD/MS/MDS) degree or a post graduate diploma were included in the study.

\section{Statistical methods}

Descriptive statistics like mean and standard deviation along with frequencies and percentages were used to analyze data. Frequencies and percentages were reported as (n, \%). Statistical tests like t-test and $\mathrm{Chi}^{2}$ tests were used to examine group differences. Statistical significance was set at $\mathrm{p}<0.05$.

\section{Results}

\section{Medication adherence monitoring practices}

Sixty-two (62\%) participants routinely monitor all patients for medication adherence while $25(25 \%)$ participants monitor a subset of patients for the same. Thirteen participants (13\%) do not monitor any patient for medication adherence. Most participants (79, 79\%) recognized themselves as responsible for medication adherence monitoring. Other health care providers recognized as currently responsible for medication adherence monitoring included nurses $(18,18 \%)$, relatives $(4,4 \%)$, nutritionists $(1,1 \%)$, social workers $(1,1 \%)$, \& pharmacists $(1,1 \%)$ while 3 participants $(3 \%)$ recognized no one specifically responsible for medication adherence monitoring. Seventy-four participants $(74 \%)$ monitored for medication adherence at every visit, three (3\%) monitored once a year and twenty-three $(23 \%)$ responded that it depended on the patient. On scale of 1 to 10 , clinicians rated their adherence monitoring practices as an average 7.01 \pm 1.81 (Mean \pm SD) with minimum being 1 and maximum being 10 .

\section{Frequency of medication adherence monitoring practices}

Responses to 10 sub-questions related to frequencies of strategies used to monitor adherence are summarized in Table 1.

The strategy most commonly classified as used "all the time" included asking patients about taking medications regularly $(72,72 \%)$. The strategy most commonly classified as "not at all" used included using electronic monitoring to assess non-adherence $(73,73 \%)$. The strategy most commonly classified as "would like to but not feasible" included monitoring blood levels of the drug to assess adherence $(20,20 \%)$.

\section{Effectiveness of medication adherence monitoring practices}

Responses to 10 sub-questions related to perceived effectiveness of strategies used to monitor adherence are summarized in Table 2.

The strategy most commonly classified as "largely" effective included asking patients about problems or difficulties taking prescribed medications $(55,55 \%)$. The strategy most commonly classified as "not at all" effective included using electronic monitoring $(61,61 \%)$.

Table 1: Frequency of adherence monitoring strategies $(\mathrm{n}=100)$

\begin{tabular}{|c|l|c|c|c|c|}
\hline S. No. & $\begin{array}{l}\text { Strategy for monitoring medication } \\
\text { adherence }\end{array}$ & \multicolumn{2}{l|}{ Number n (\%) of "Yes" Answers Indicating frequency } \\
\hline & & Not at all & Sometimes & All the time & $\begin{array}{c}\text { Would like to but } \\
\text { not feasible }\end{array}$ \\
\hline & $\begin{array}{l}\text { Assess understanding of patients on how to } \\
\text { take the prescribed medications using } \\
\text { feedback }\end{array}$ & $3(3)$ & $37(37)$ & $47(47)$ & $13(13)$ \\
\hline 2 & $\begin{array}{l}\text { Ask patients whether they feel better or } \\
\text { worse after taking the prescribed medication }\end{array}$ & $6(6)$ & $33(33)$ & $51(51)$ & $10(10)$ \\
\hline 3 & $\begin{array}{l}\text { Assess patients' own beliefs, knowledge, } \\
\text { and expectations about treatment }\end{array}$ & $9(9)$ & $59(59)$ & $19(19)$ & $13(13)$ \\
\hline 4 & Ask patients about financial concerns & $6(6)$ & $32(32)$ & $53(53)$ & $9(9)$ \\
\hline 5 & $\begin{array}{l}\text { Ask patient about taking medications } \\
\text { regularly }\end{array}$ & $0(0)$ & $28(28)$ & $72(72)$ & $0(0)$ \\
\hline 6 & $\begin{array}{l}\text { Ask patients about any problems or } \\
\text { difficulties taking prescribed medications }\end{array}$ & $7(7)$ & $42(42)$ & $46(46)$ & $5(5)$ \\
\hline 7 & Use electronic monitoring & $73(73)$ & $7(7)$ & $6(6)$ & $14(14)$ \\
\hline 8 & Monitor blood levels & $59(59)$ & $21(21)$ & $0(0)$ & $20(20)$ \\
\hline 9 & Review of medication journal/diary & $43(43)$ & $44(44)$ & $3(3)$ & $10(10)$ \\
\hline 10 & Count tablets during follow up & $20(20)$ & $43(43)$ & $19(19)$ & $18(18)$ \\
\hline
\end{tabular}


Table 2: Effectiveness of adherence monitoring strategies $(n=100)$

\begin{tabular}{|c|c|c|c|c|}
\hline \multirow[t]{2}{*}{ S. No. } & \multirow[t]{2}{*}{ Strategy for monitoring medication adherence } & \multicolumn{3}{|c|}{$\begin{array}{c}\text { Number n (\%) of "Yes" Answers Indicating } \\
\text { effectiveness }\end{array}$} \\
\hline & & Not at all & Somewhat & Largely \\
\hline 1 & $\begin{array}{l}\text { Assess understanding of patients on how to take } \\
\text { the prescribed medications using feedback }\end{array}$ & $3(3)$ & $49(49)$ & $48(48)$ \\
\hline 2 & $\begin{array}{l}\text { Ask patients whether they feel better or worse } \\
\text { after taking the prescribed medication }\end{array}$ & $6(6)$ & $58(58)$ & $36(36)$ \\
\hline 3 & $\begin{array}{l}\text { Assess patients' own beliefs, knowledge, and } \\
\text { expectations about treatment }\end{array}$ & $16(16)$ & $61(61)$ & $23(23)$ \\
\hline 4 & Ask patients about financial concerns & $17(17)$ & $34(34)$ & $49(49)$ \\
\hline 5 & Ask patient about taking medications regularly & $3(3)$ & $45(45)$ & $52(52)$ \\
\hline 6 & $\begin{array}{l}\text { Ask patients about any problems or difficulties } \\
\text { taking prescribed medications }\end{array}$ & $13(13)$ & $32(32)$ & $55(55)$ \\
\hline 7 & Use electronic monitoring & $61(61)$ & $27(27)$ & $12(12)$ \\
\hline 8 & Monitor blood levels & $47(47)$ & $41(41)$ & $12(12)$ \\
\hline 9 & Review of medication journal/diary & $33(33)$ & $51(51)$ & $16(16)$ \\
\hline 10 & Count tablets during follow up & $20(20)$ & $54(54)$ & $26(26)$ \\
\hline
\end{tabular}

\section{Perceptions of clinicians in regards to medication adherence monitoring}

Participants believed that the health care provider who should be tasked with monitoring patients for medication adherence should be the clinician $(55,55 \%)$, nurse $(45,45 \%)$, pharmacist $(33,33 \%)$, social worker $(30,30 \%)$, relative/patient $(9,9 \%)$ and nutritionist $(3,3 \%)$. Twelve participants (12\%) believed that no one should be responsible for monitoring medication adherence. Fifty-nine (59\%) participants believed medication adherence monitoring should be done at every visit while forty-one $(41 \%)$ participants believed that it should depend on the patient. None of the participants believed that adherence should not be monitored at all, once a year or twice a year. In regards to importance of routine adherence monitoring in clinical practice, $73(73 \%)$ participants believed that it was "largely" important while 27 (27\%) participants believed that it was "somewhat" important.

\section{Impact of clinical experience, training on medication adherence monitoring practices \\ Clinical experience}

Participants with more than 10 years experience estimated their patients' adherence rates to be approximately $71.18 \%$ while those with less than 10 years experience estimated their patients' adherence rates to be approximately $66.72 \%$. On a scale from 1 to 10 , participants with more than 10 years experience estimated their adherence monitoring practices to be $8.22 \pm 1.31$ (Mean $\pm \mathrm{SD}$ ) while those with less than 10 years experience estimated the same to be $6.66 \pm 1.78$ $(\mathrm{p}<0.05)$. Approximately $73.91 \%$ participants with greater than 10 years clinical experience and $31.17 \%$ of those with less than 10 years experience rated their adherence monitoring practices as 8 or above on a scale of 1 to 10 . $(\mathrm{p}=0.000255)$.

\section{Training}

Participants with some form of training in medication adherence estimated their patients' adherence rates to be approximately $70.00 \%$ while those with no training estimated their patients' adherence rates to be approximately $67.30 \%$. On a scale from 1 to 10 , participants with some training estimated their adherence monitoring practices to be $7.78 \pm 1.48$ (Mean $\pm \mathrm{SD}$ ) while those with no training estimated the same to be $6.84 \pm 1.84(\mathrm{p}=0.013)$.

\section{Discussion}

Despite the fact that previous studies have established the negative effects of poor adherence on morbidity and mortality as well as costs to the healthcare system, medication adherence remains a highly prevalent problem. ${ }^{2-}$ ${ }^{6}$ It is hypothesized that awareness about non-adherence is low among clinicians. ${ }^{2}$ Due to this, a number of cases of low adherence go undetected in clinical practice. Hence, we have conducted this study to examine the current adherence monitoring practices, their perceived effectiveness, and perceptions of clinicians in our institute in relation to medication adherence monitoring.

\section{Medication adherence monitoring -current practices}

Sixty two percent participants routinely monitor all patients for medication adherence while $25 \%$ participants monitor a subset of patients. Often, clinicians will monitor a subset of patients only, due to lack of time and high patient load and in patients prescribed drugs with low therapeutic index. Patients with chronic disease or infectious diseases are also more likely to need intense adherence monitoring. In the study conducted by Maddux et al. approximately 56\% participants monitored all patients while approximately $44 \%$ monitored a subset. ${ }^{9}$ Thirteen participants $(13 \%)$ in our study did not monitor any patient for medication adherence. The relatively high percentage $(62.5 \%)$ of participants who monitor all patients in our study may be due to the fact that most participants report use on methods that require few 
resources. If resource intensive methods are adopted, it is possible that more clinicians will start monitoring only a subset of patients.

Most participants (79\%) recognized themselves as responsible for medication adherence monitoring. A similar percentage of clinicians $(74.7 \%)$ identified themselves as being tasked with medication adherence in the study by Maddux et al. Other team members recognized as currently responsible for medication adherence monitoring included nurses $(18 \%)$ which is a low number compared to other studies. ${ }^{8,9}$ This percentage of team members other than clinicians being tasked with medication adherence monitoring is much less than those studies. ${ }^{8,9}$ This maybe due to the fact that most of the burden of health care delivery falls on the clinician in our system.

Participants rated their adherence monitoring practices fairly high at an average of approximately 7 on a scale of 1 to 10 . This would indicate that most participants use some strategy to monitor medication adherence among patients in their clinical practice.

The method most used to monitor medication adherence was asking patient about taking medications regularly $(72 \%)$. This is similar to another study by Berben et al $(61 \%) .{ }^{8}$ This technique is commonly used as it is easy and feasible. ${ }^{8}$ Another method used frequently in our study was asking patients about financial constraints that are likely to be a barrier to adherence (53\%). This method is an indirect way of estimating and predicting the likelihood of a patient adhering to their medication. As all clinicians in our sample identified their patients as being from lower to middle socio economic classes, cost becomes an important factor. Patients who can't afford the prescribed drug are less likely to be adherent. The strategy most commonly classified as "not at all" used was electronic monitoring to assess non-adherence $(73,73 \%)$. This was also reported to be rarely used $(0.6 \%)$ in the study by Maddux et al. ${ }^{9}$

Fifty nine percent of participants'classified monitoring blood levels of the drug as not being used at all. This is an objective measure of medication adherence and used in 50\% of patients in the study by Maddux et al. ${ }^{9}$ The relative low percentage of respondents using this method in our sample may be due to cost considerations. Additionally, several hospitals may not be performing drug level tests. This may be the reason why $20 \%$ participants rated monitoring blood levels as a measure they would like to do but do not find feasible. Monitoring blood levels was the method most commonly classified as "would like to use but not feasible." Another method that $18 \%$ participants classified as "would like to use but not feasible" included counting tablets during follow up. Pill counting is a simple method of measuring adherence but is subject to problems such as the patient discarding pills before the visit with health care provider. Thus pill counting is not an accurate way tomeasure medication adherence. ${ }^{3}$

Overall in our sample, methods that require fewer resources were used much more than resource intensive methods to monitor medication adherence. This may be due to a perceived lack of effectiveness or a perceived lack of feasibility.

\section{Effectiveness of current medication adherence monitoring strategies}

The strategies most commonly classified as "largely" effective included asking patients about taking medications regularly $(52 \%)$ or problems or difficulties taking the prescribed medications (55\%). However, the patient selfreport is often unreliable and such techniques may overestimate the overall medication adherence. This may be due to the fact that patients may have difficulty remembering missed doses and maylead to higher than actual adherence rates. ${ }^{9}$

Approximately, $61 \%$ perceive electronic monitoring as "not at all" effective which matches their use. Approximately 47\% perceived monitoring blood levels as "not at all" effective, which also matches their use. Despite $59 \%$ believing that monitoring blood levels would not be at all effective, $20 \%$ would like to monitor blood levels to assess non-adherence but cannot due to feasibility issues. There is no gold standard to monitor adherence. ${ }^{3,8}$ Multiple methods should be used with subjective and objective measures. ${ }^{3,8,9}$ Clinicians should be made aware of the differences between subjective and objective methods.

\section{Impact of training \& clinical experience on adherence monitoring practices}

Participants with more than 10 years experience estimated their adherence monitoring practices to be significantly higher on a scale of 1 to 10 than those with less than 10 years experience. Participants with more than 10 years experience estimated higher adherence rates than those with less than 10 years experience. This shows clinical experience significantly affects the participants grading of their own adherence monitoring practices, which leads to higher adherence rates. A study by Patel et al. also demonstrated that clinical experience affects monitoring practices positively. ${ }^{7}$

\section{Limitations}

Our sample only represents the views of clinicians working in tertiary care teaching hospital. Self-reports by clinicians, though a necessary first step, are subjective. Future studies can focus on correlating these self-reports with more objective measures. Further studies can also focus on qualitative approaches that can provide more information and understanding into the answers given by participants.

\section{Conclusion}

This study has important implications for developing optimum practices for medication adherence monitoring. Clinicians in our study seemed to be well aware of the importance of medication adherence monitoring practices. Overall, our results demonstrate the need to develop systems supporting medication adherence monitoring in all hospitals. 


\section{Acknowledgment}

The study was done as part of the Indian Council of Medical Research-Short Term Studentship (ICMR-STS) Programme 2019. We acknowledge and thank the Indian Council of Medical Research (ICMR) for supporting and sponsoring this research.

\section{Financial Support and Sponsorship}

This study was financially supported by the ICMR STS Program 2019.

\section{Conflicts of Interest}

There are no conflicts of interest.

\section{Ethical Approval}

The study was approved by the Institutional Ethics Committee.

\section{Contributors}

All authors were involved in conceiving, organizing the study, analysis and interpretation of results and drafting the manuscript. Data collection was done by Vansh Nagrani. Vansh Nagrani was the principal investigator and Nehal Shah was the guide and co- principal investigator.

\section{References}

1. Sabaté E. Adherence to long-term therapies: evidence for action. World Health Organization; 2003.

2. Kleinsinger $F$. The Unmet Challenge of Medication Nonadherence. Perm J. 2018; 22:18-33.
3. Osterberg L, Blaschke T. Adherence to medication. $N$ Engl $J$ Med. 2005;353(5):487-97.

4. Wu JR, Moser DK. Medication Adherence Mediates the Relationship Between Heart Failure Symptoms and Cardiac Event-Free Survival in Patients With Heart Failure. $J$ Cardiovasc Nurs. 2018;33(1):40-6.

5. Saag KG, Bhatia S, Mugavero MJ, Singh JA. Taking an Interdisciplinary Approach to Understanding and Improving Medication Adherence. J Gen Intern Med. 2018;33(2):136-8.

6. Nieuwlaat R, Wilczynski N, Navarro T. Interventions for enhancing medication adherence. Cochrane Database Syst Rev. 2014;(11):CD000011.

7. Patel UD, Davis MM. Physicians' attitudes and practices regarding adherence to medical regimens by patients with chronic illness. Clin Pediatr (Phila). 2006;45(5):439-45.

8. Berben L, Dobbels F, Kugler C, Russell CL, De Geest S. Interventions used by health care professionals to enhance medication adherence in transplant patients: a survey of current clinical practice. Prog Transplant. 2011;21(4):322-31.

9. Maddux MH, Ricks S, Bass JA, Daniel JF, Carpenter E, Radford K. Practice survey: adherence monitoring and intervention in pediatric gastroenterology and hepatology. Ther Clin Risk Manag. 2018;14:1227-34.

How to cite this article: Shah NA, Nagrani V, Patel V. A questionairre based study evaluating current medication adherence monitoring practice in a tertiary care teaching hospital. Indian J Pharm Pharmacol 2020;7(1):10-4. 